\title{
CABERGOLINE THERAPY IN CLINICALLY NONFUNCTIONING PITUTIARY ADENOMA: RESULTS OF A CLINICAL TRIAL
}

Rafael Loch Batista', Malebranche Berardo Carneiro da Cunha Neto', Nina Rosa Castro Musolino', Valter Angelo Cescato', Clarissa Groberio Borba', Gilberto Ochman da Silva1, Ericka Trarbach²

1 Neuroendocrine Unit, Neurosurgical Unit, Universidade de São Paulo

${ }^{2}$ Molecular Biology Laboratory - LIM 25 - Medical College, Universidade de São Paulo

INTRODUCTION: Nonfunctioning pituitary adenomas (NPA) are prevalent neoplams from pituitary. The best treatment is the surgical approach but residual tumours are common. For residual tumours the options are radiotherapy (RT) and re-surgery. There are no significant studies testing drugs in NPA. Due to the fact that these tumors express dopamine D2 receptors, dopamine agonists that may be useful in patients with NPA.

OBJECTIVE: To evaluate the useful of cabergoline in NFPA patients with residual tumor after transsphenoidal (TS) surgery, in reducing and/or maintain a stable tumor rest.

MATERIAL AND METHODS: We selected 145 patients with residual tumor after surgery and imunohistochemical confirmation. Hormonal hypersecretion was excluded in all of them. RMI was performed six months after surgery to confirm residual tumour. After this time, the patients were randomized alternately in two groups: for cabergoline (Group $A ; n=$ 74 ) or just clinical observation (Group $B ; n=71$ ). RMI was performed at baseline and each 6 months by 24 months. Tumour residual volume and immunohistochemical expression was compared after 12 and 24 months. The tumour volume was calculated according by Lundin and Peterson $\left(\mathrm{cm}^{3}\right)$ and by a software program (Osirix). Tumor shrinkage $>25 \%$ of baseline was considered significant. Any increase in tumor volume during follow-up was considered relevant. Ecocardiography was performed as a safety measure.
RESULTS: Group A (CAB) showed tumor reduction in $20,89 \%$ of patients and tumor increase in $7,46 \%$. Stabilization of residual tumour was observed in $71,64 \%$. In group B, reduction was observed in $4.91 \%$, tumour progression in $16,39 \%$ and no changes in $78,68 \%$. Significant differences was observed in tumor reduction $(A>B ; p<0.01)$ and tumor progression ( $>A ; p .02)$. Stabilization was similar between two groups $(p=0,4)$. No echocardiography alterations was observed between baseline, 12 and 24 months (Ao, Pulm, Mitral, Tric: $p=$ NS). Imunohistochemical profile was not different between two groups and not related to tumor progression or reduction.
Cabergoline $n=74$

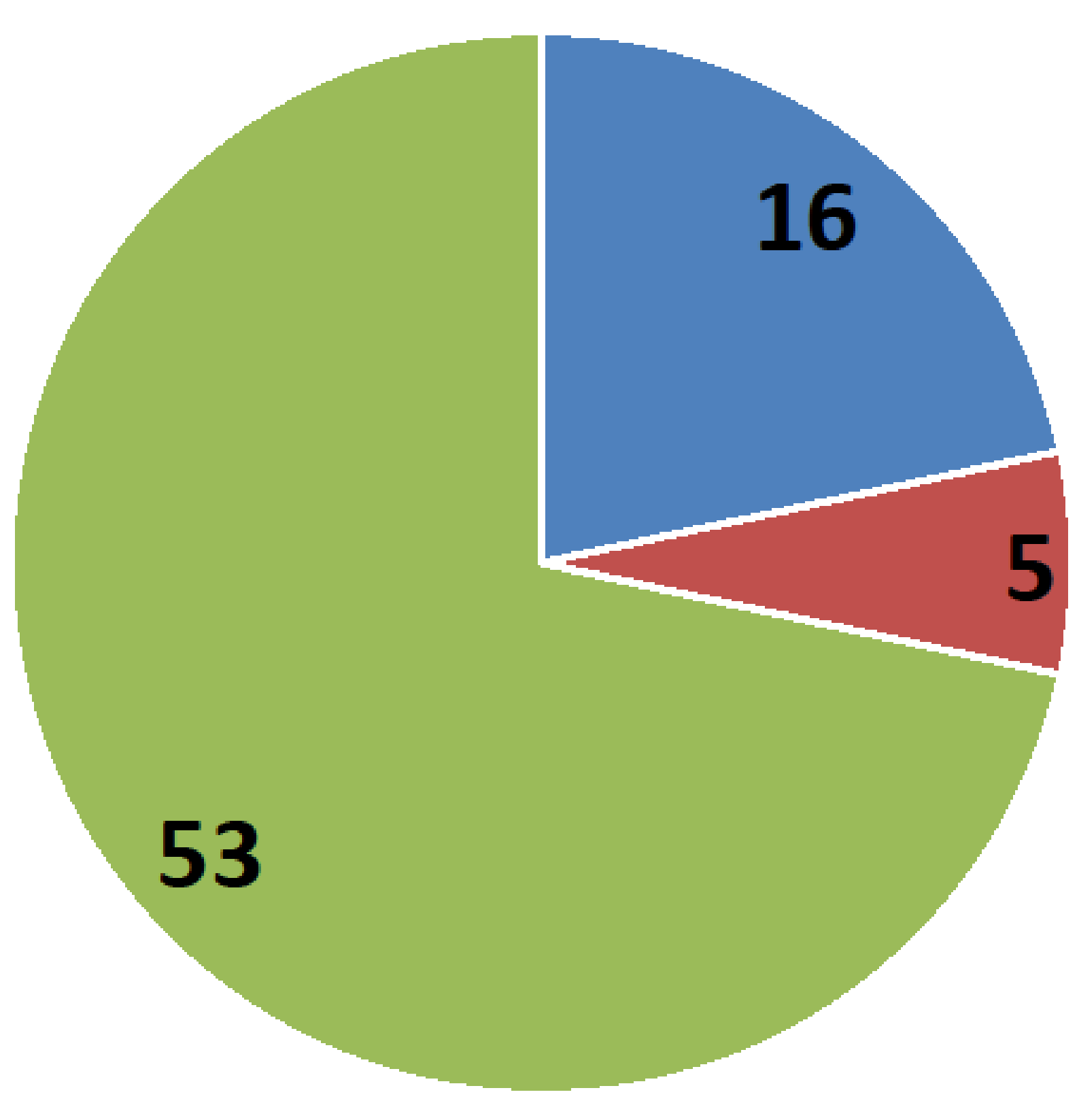

- Reduction $\quad$ Increase

- Stabilization
Control $n=71$

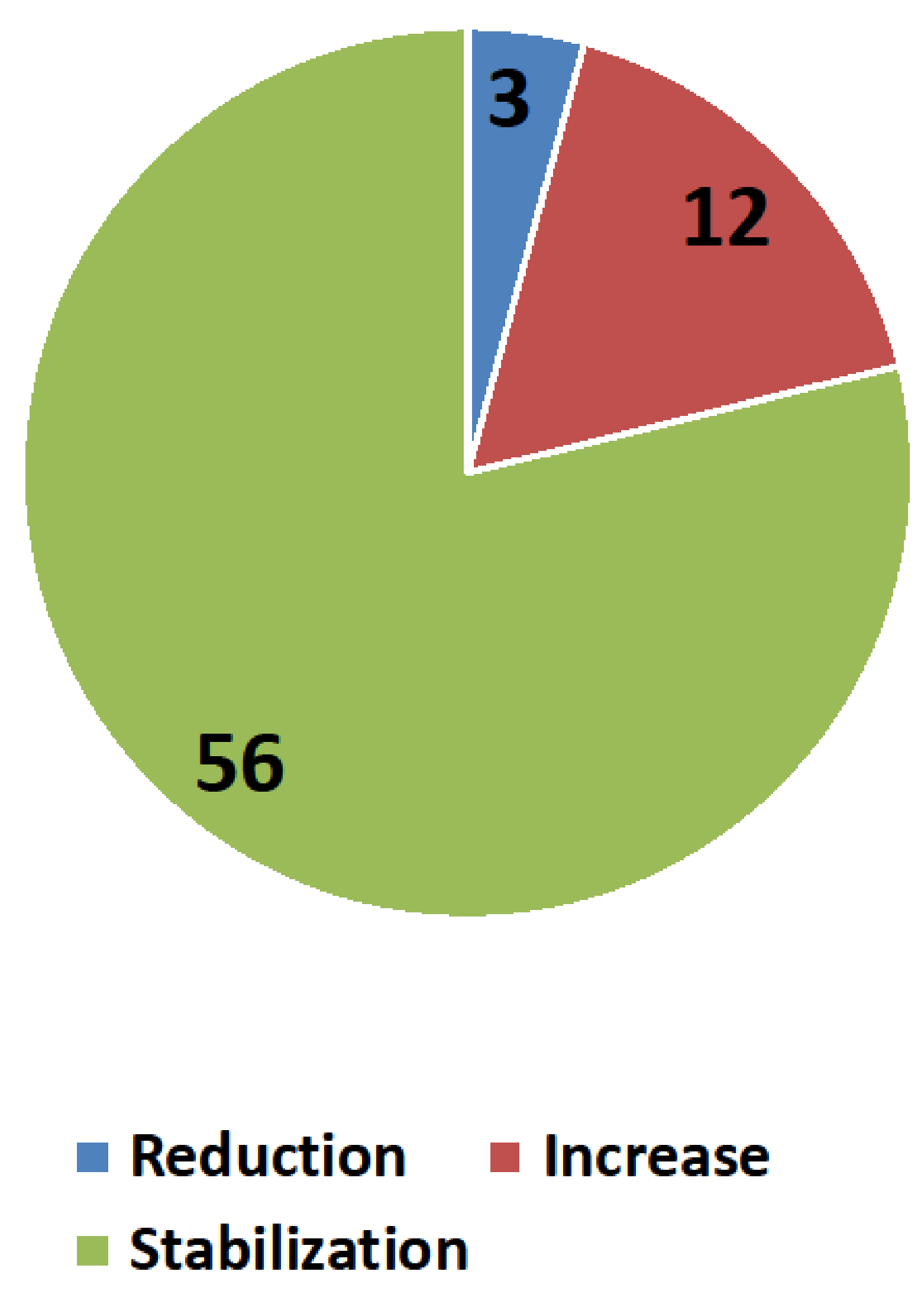

CONCLUSION: Cabergoline was a effective alternative to radiotheraphy or resurgery in NPA. Residual tumour reduction was observed in $1 / 5$ of patients. The immunohistochemical profile is not predictive of response to cabergoline. Cabergoline did not show cause changes in heart valves in this study. 